\title{
Determination of sequential mutation accumulation in pancreas and bile duct brushing cytology
}

\author{
Odeta Lapkus ${ }^{1}$, Olguta Gologan ${ }^{1}$, Yulin Liu ${ }^{1}$, Patricia A Swalsky², Matthew M Wilson ${ }^{2}$, \\ Sydney D Finkelstein ${ }^{2}$ and Jan F Silverman ${ }^{1}$ \\ ${ }^{1}$ Department of Pathology and Laboratory Medicine, Drexel University College of Medicine, Allegheny \\ General Hospital, Pittsburgh, PA, USA and ${ }^{2}$ RedPath Integrated Pathology Inc, Pittsburgh, PA, USA
}

\begin{abstract}
Neoplastic progression is characterized by clonal expansion of tumor cells associated with accumulation of mutational damage. The timing of mutation acquisition could be of value in distinguishing preneoplastic conditions from early and advanced cancer as well as characterizing tumor aggressiveness and treatment response. Using quantitative methods applied to microdissected cell clusters selected according to cytomorphologic features, we sought to demonstrate the feasibility and efficacy for determining the time and course of mutation accumulation in pancreatobiliary cytology specimens. In all, 40 pancreatic duct and 21 biliary brushing cytology specimens were retrieved from the cytology database. Xylene-resistant markings were placed on the slide underside and coverslips removed. Clusters of benign, atypical and malignant cells were manually microdissected and DNA extracted. Mutations (allelic imbalance) (loss of heterozygosity) were quantitatively determined for a broad panel of 15 markers (1p, 3p, 5q, 9p, 10q, 17p, 17q, 21q, 22q) as well as point mutation in $K$-ras-2 using PCR/capillary electrophoresis. Time course was based on earlier mutations having a higher proportion of mutant DNA for a particular marker. The descending frequency of detectable mutational involvement in pancreatic cytology was $K$-ras-2 point mutation (58\%), 3p25-26 and 17q21 (35\%), 5q23 (33\%), $1 \mathrm{p} 36(28 \%)$, followed by the remaining molecular markers. The descending frequency of mutational content in bile duct cytology was $17 \mathrm{p} 13,1 \mathrm{p} 36,3 \mathrm{p} 25-26$, and $5 \mathrm{q} 23$ followed by remaining molecular markers. $K$-ras-2 point mutation was not seen in bile duct specimens. While there was overlap in the spectrum of mutational markers in pancreatic duct and biliary brushing cytology, the temporal profile was significantly different $(\boldsymbol{P}<0.001)$. Pancreatic and biliary neoplasia progression involves distinct subset of accumulated defined mutations. Determination of timing of the mutational damage in cytologic material could be incorporated in the work-up and help in making a more definitive diagnosis of malignancy in pancreatobiliary cytology specimens. Modern Pathology (2006) 19, 907-913. doi:10.1038/modpathol.3800545; published online 28 April 2006
\end{abstract}

Keywords: cytology; pancreaticobiliary brushing cytology; mutation; loss of heterozygosity; temporal sequence of mutations

Cytologic evaluation is increasingly being used as a first step in cancer detection, diagnosis and treatment planning. ${ }^{1-3}$ However, pancreaticobiliary cytology can be challenging, since the differential diagnosis includes reactive states (pancreatic pseudocyst, pancreatitis, biliary stricture), low-grade neoplastic lesions (mucinous cystic neoplasms, intraductal papillary mucinous neoplasia) and malignant tumors (pancreatic adenocarcinoma, cho-

Correspondence: Dr JF Silverman, MD, Department of Pathology, Drexel University College of Medicine, Allegheny General Hospital, 320 East North Street, Pittsburgh, PA 15212, USA.

E-mail: jsilverm@wphas.org

Received 28 July 2005; revised and accepted 17 November 2005; published online 28 April 2006 langiocarcinoma). ${ }^{4-6}$ Although cytologic diagnosis can be definitive and specific, in over $50 \%$ of such specimens the diagnosis is indeterminate. ${ }^{5,6}$ With an atypical but indeterminate diagnosis, surgery may be performed for nonmalignant disease based on clinical and imaging features. Alternatively, indeterminate cytology may lead to conservative measures with surveillance and repeat examinations for a malignant condition. Therefore, in some cases, an indeterminate diagnosis may contribute to miss early cancer when surgery would be effective and later only palliation may be feasible. Owing to occasional limitations of cytology, we investigated whether molecular methods can provide additional information that can reduce the rate of indeterminate diagnosis. 
The transformation from a benign to malignant phenotype is associated with sequential acquisition of mutational alterations. ${ }^{7-9}$ Various tumor-suppressor genes are lost and oncogenes activated at different stages of tumor development. The time when each mutation occur may determine the differences in tumor biological behavior, can influence the rate of subsequent mutation acquisition, characterize phenotypic variability of the cancer type and help to distinguish preneoplastic conditions from cancer. ${ }^{7-11}$ Therefore, it is reasonable to believe that two neoplasms with essentially the same mutational profile but acquired at a different time should behave in a different manner with respect to neoplastic features. In addition, molecular findings may assume greater importance as gene specific therapy is introduced and increasingly used for cancer treatment.

Cancer arises via a multistep process of mutational accumulation that is responsible for phenotypic expression of biological properties, such as tumor invasiveness, metastatic spread and treatment responsiveness. ${ }^{7-9}$ Acquired mutations that confer significant growth advantage will induce clonal expansion of phenotypically more aggressive tumor cells. ${ }^{12-14}$ This will result in increasing numbers of neoplastic cells bearing the mutation, which may be appreciated microscopically by increased cellular atypia. Mutational change that is not associated with positive growth advantage will be diluted as a genotypic alteration in the overall pool of proliferating tumor cells. It is important to distinguish between acquired mutations that induce clonal expansion vs acquired mutations occurring in the background and not associated with significant proliferative features. ${ }^{15,16}$

Mutations which are present in a large proportion of cells more likely have been acquired earlier then mutations present in lesser numbers of cells with more localized distribution. ${ }^{10,11,17}$ These molecular changes have not yet been previously described in cytology specimens where such information could greatly impact patient management. Our objective was to extend molecular studies to cytology specimens to determine the feasibility and effectiveness of mutation temporal profile determination in the anticipation that such information can potentially be used to render a more definitive cytologic diagnosis for pancreatic and biliary neoplasms.

\section{Materials and methods}

A total of 40 pancreatic and 21 biliary duct brushing cytology specimens were retrieved from Allegheny General Hospital cytology archives for 1999-2004. Xylene-resistant markings were placed on the slide underside representing cellular targets for integrated molecular analysis. Clusters of normal $(n=10)$, atypical $(n=20)$ and malignant $(n=10)$ appearing cells in papanicolaou-stained smears were manually microdissected under stereomicroscopic observation. ${ }^{18,19}$ Mutational analysis was based on polymerase chain amplification reaction (PCR) for a broad panel of genomic markers associated with tumor suppressor gene loss and oncogene activation commonly involved in human pancreatic and biliary carcinogenesis. ${ }^{20-22}$

Aliquots $(1 \mu \mathrm{l})$ of the microdissected cells were used in individual genotyping reactions for a panel of markers consisting of $K$-ras-2 exon 1 for direct DNA sequencing and 15 allelic imbalance microsatellite markers situated at 1p34-36 (two markers), 3p25-26 (two markers), 5p23 (two markers), 9p2123 (two markers), 10q23 (two markers), 17p13 (two markers), 17q21 (one marker), 21q23 (one marker) and 22q12 (one marker). The corresponding genes for the markers used are as follows: 1p markers: $C M M$; 3p markers: VHL; 5q markers: $A P C$; 9p markers: CDKN2A; 10q markers: PTENN; 17p markers: TP53; 17q marker: NME1. PCR amplification was designed to generate amplicons measuring $<200$ base pairs long using synthetic oligonucleotide primers flanking each microsatellite. Oligonucleotide primers were created with $5^{\prime}$ fluorescent moieties (FAM, HEX, NED) suitable for automated fragment analysis. The PCR products were analyzed by capillary electrophoresis on an ABI 3100 according to manufacturer's instructions (Applied Biosystems, Foster City, CA, USA). ${ }^{23}$ Allele peak heights and lengths were used to define the presence or absence of allelic imbalance (ie, loss of heterozygosity) for a given sample. Allelic imbalance was reported when the ratio of polymorphic allelic bands for a particular marker was beyond 95\% confidence limits for the variation in peak heights for individual allele pairings derived from analysis using non-neoplastic specimen samples in preliminary studies. In general, this value was below 0.5 or above 2.0. DNA sequencing of $K$-ras-2 exon 1 PCR amplified DNA was used to search for and characterize point mutations in codons 12 and 13 . The peak heights of normal and mutant bases were used to determine the proportion of mutated and nonmutated cells in each microdissected sample. Replication was performed on multiple samples where sufficient numbers of cells were available and on individual markers to validate the genotyping results. The lower threshold for detection of significant allelic imbalance (LOH) was approximately $40 \%$ of microdissected cells whereas the threshold for point mutation detection was approximately $20 \%$.

When the average value for normal sample allele peak height ratios is 1.00 , allele ratios of 2.0 or 0.5 corresponded to $50 \%$ of the microdissected cells bearing allelic imbalance for that particular marker. The deviation from ideal normal ratio of 1.0 indicated which specific allele was affected. Based on a model of clonal expansion, the order of mutation acquisition could then be arranged in a sequence reflecting the proportion of cells affected 
by specific microsatellite marker loss and point mutation. Markers displaying more extreme ratios are considered to have been acquired earlier. When two or more mutations, K-ras-2 and/or allelic imbalance mutations, were detected, their time course of accumulation was determined by the proportion of total DNA manifesting the alteration. In the case of $K$-ras-2 oncogene point mutation, involvement of $100 \%$ of cells was considered to be present when the density of the mutated base on sequencing autoradiography was equal to or greater than the normal sequence base pair.

\section{Results}

Cytology preparations generally provided a small but sufficient number of representative cells for molecular analysis. Individual clusters of target cells were microdissected and collected for broad panel genotyping. Every effort was made to replicate the analysis both at the microdissected target level and at the individual marker level when the specimen was limited.

The ratio of allelic peak heights is a quantitative measure of the proportion of mutated and nonmutated cells in the microdissected cytology sample (Figure 1). It should be noted that the nonmutated cells for a particular marker are composed of two groups: non-neoplastic cells included in the microdissection and neoplastic cells that do not bear that specific mutational change. The first is a fixed value for each microdissected sample and present in all mutational analyses for that sample. This normal cellular component enabled accurate determination of the status for each allelic imbalance marker and did not account for $>5 \%$ of total microdissected cells.

The second component of nonmutated DNA associated with neoplastic cells reflects the timing of specific mutation acquisition in that mutational damage acquired early and causally linked to clonal expansion would be present in the greatest proportion of neoplastic cells (Figure 1). Since each mutational analysis, uses an equivalent amount of total sample DNA, significant differences in imbalance peak height ratios could be directly linked to the temporal pattern of specific marker mutation (Figure 1).

In the case of point mutational change, a similar analysis could be carried out (Figure 2). The ratio of peak heights of the normal and mutant bases correlated with the proportion of mutated and nonmutated cells (Figure 2).

The complete data for all patient specimens is shown in Table 1 . In addition to discrimination of normal and mutated status, markers showing mutational change were separated according to specific allele affected by imbalance, specific form of K-ras-2 point mutation and proportion of microdissected cells affected by mutational change. As all values are

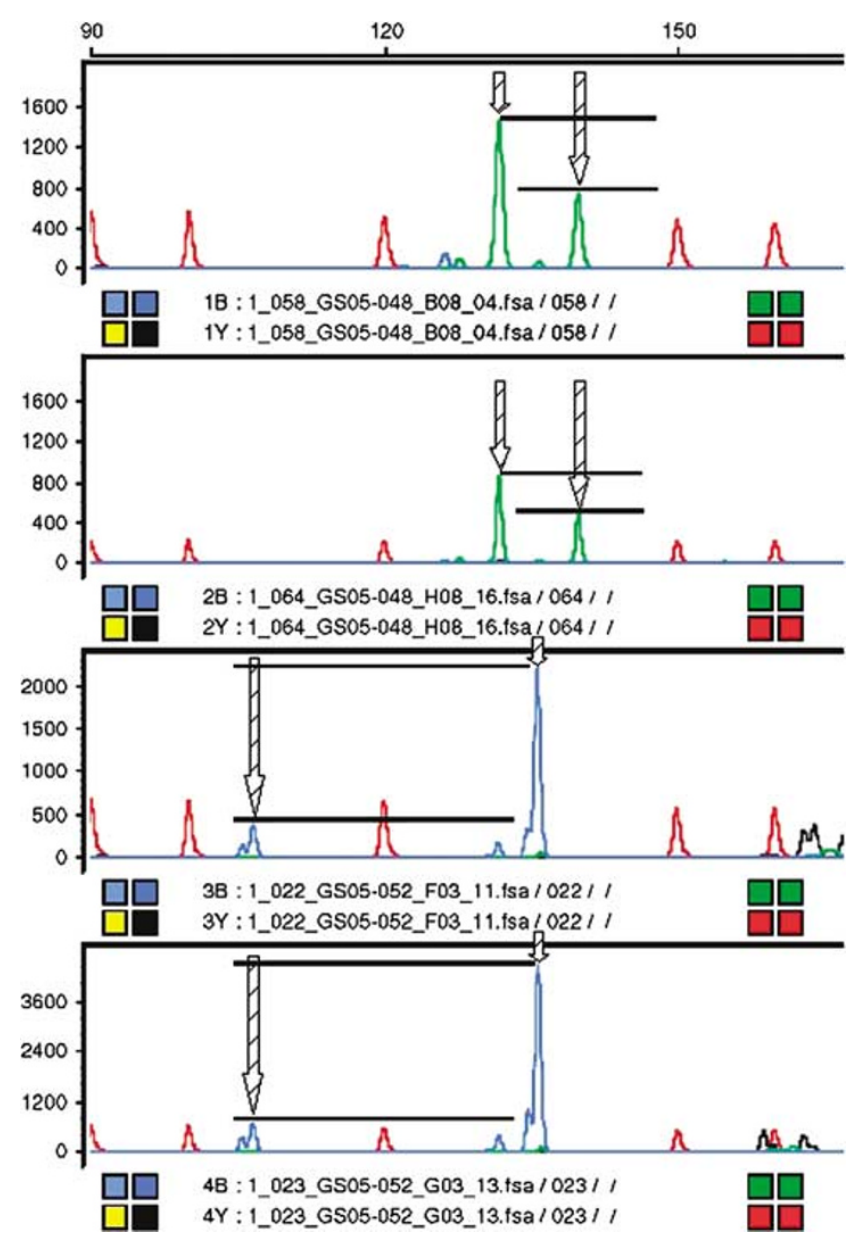

Figure 1 Using equivalent aliquots of microdissected sample DNA, certain markers show lower degrees of significant imbalance (LOH) (upper pair of samples) than other markers, which exhibit major imbalance (lower pair of samples). The quantitative degree of imbalance is indicated by arrows and horizontal lines reflecting difference in peak heights. This enables a determination of the temporal profile of mutation acquisition in which earlier mutations manifested more extreme degrees of imbalance.

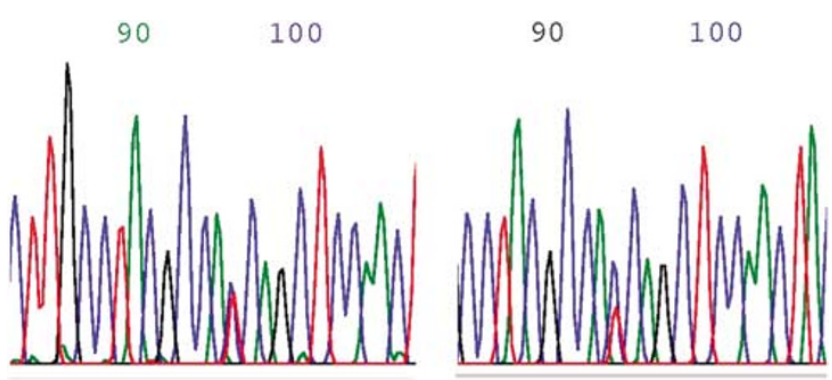

Figure 2 The same relationship between relative differences in peak height and temporal mutation acquisition apply to point mutation determination as for K-ras-2 exon 1. In the upper panel, the height of the normal band and the mutant band is nearly equivalent indicating that $100 \%$ of the microdissected cells are heterozygous for the oncogenic point mutation. In the lower panel, the mutant band is approximately $60 \%$ the height of the normal band indicating that $60 \%$ of the microdissected cells have acquired the point mutation. 
Table 1 Complete data set for temporal analysis

\begin{tabular}{|c|c|c|c|c|c|c|c|c|c|c|c|c|c|c|c|c|}
\hline & & $1 \mathrm{p} 36$ & 1p 36 & $3 p 25$ & $3 \mathrm{p} 25$ & $5 q 23$ & $5 q 23$ & $9 \mathrm{p} 21$ & $9 \mathrm{p} 21$ & $10 \mathrm{q} 23$ & $10 \mathrm{q} 23$ & $17 \mathrm{p} 13$ & $17 p 13$ & $17 q 23$ & $21 q 23$ & $22 q 12$ \\
\hline & \multirow{2}{*}{ KRAS } & D1S & D1S & D3S & D3S & D5S & D5S & D9S & D9S & D10S & D10S & D17S & D17S & D17S & D21S & D22S \\
\hline & & 1193 & 407 & 1539 & 2303 & 592 & 615 & 251 & 254 & 1173 & 520 & 1289 & 974 & 1161 & 144 & 532 \\
\hline \multicolumn{17}{|c|}{ Pancreas } \\
\hline 1 & NO MUT & NI & $\mathrm{NO}$ LOH & NO LOH & $\mathrm{NI}$ & NO LOH & NO LOH & NO LOH & $\mathrm{NI}$ & $\mathrm{NO}$ LOH & $66 \%$ & NO LOH & NI & $\mathrm{NO} \mathrm{LOH}$ & $\mathrm{NO} \mathrm{LOH}$ & NO LOH \\
\hline 2 & $12 \cdot R$ & $\mathrm{NO}$ LOH & NI & NO LOH & NO LOH & NO LOH & NO LOH & NI & NOLOH & $\mathrm{NO} \mathrm{LOH}$ & $\mathrm{NOLOH}$ & $\mathrm{NI}$ & NI & $\mathrm{NO} \mathrm{LOH}$ & $\mathrm{NOLOH}$ & NO LOH \\
\hline 2 & $12 \cdot R$ & NO LOH & NI & NO LOH & NOLOH & NO LOH & NOLOH & NI & NOLOH & NOLOH & NOLOH & $\mathrm{NI}$ & NI & $\mathrm{NO} \mathrm{LOH}$ & $\mathrm{NOLOH}$ & NOLOH \\
\hline 3 & 12-D & NO LOH & $\mathrm{NI}$ & NO LOH & NOLOH & $51 \%$ & NOLOH & NI & NOLOH & NO LOH & $\mathrm{NO} \mathrm{LOH}$ & NO LOH & NOLOH & $\mathrm{NI}$ & $\mathrm{NOLOH}$ & NOLOH \\
\hline 3 & 12-D & $\mathrm{NO}$ LOH & NI & NOLOH & NO LOH & $54 \%$ & NO LOH & NI & $\mathrm{NO} \mathrm{LOH}$ & $\mathrm{NO}$ LOH & $\mathrm{NO}$ LOH & NO LOH & NOLOH & NI & $\mathrm{NO}$ LOH & $58 \%$ \\
\hline 4 & $12 \cdot R$ & $77 \%$ & NOLOH & $\mathrm{NI}$ & $61 \%$ & NI & NOLOH & NOLOH & $\mathrm{NOLOH}$ & NI & $\mathrm{NO}$ LOH & $88 \%$ & NO LOH & $\mathrm{NO}$ LOH & $\mathrm{NO}$ LOH & $\mathrm{NOLOH}$ \\
\hline 4 & $12 \cdot R$ & $71 \%$ & $\mathrm{NO}$ LOH & $\mathrm{NI}$ & $59 \%$ & $\mathrm{NI}$ & NO LOH & NO LOH & NO LOH & $\mathrm{NI}$ & $\mathrm{NO}$ LOH & $88 \%$ & NO LOH & $\mathrm{NO} \mathrm{LOH}$ & $\mathrm{NO} \mathrm{LOH}$ & NO LOH \\
\hline 5 & $12 \cdot \mathrm{C}$ & $\mathrm{NI}$ & $91 \%$ & NO LOH & NOLOH & $89 \%$ & $86 \%$ & NOLOH & NI & NO LOH & $\mathrm{NO}$ LOH & $\mathrm{NI}$ & $79 \%$ & $76 \%$ & NI & NO LOH \\
\hline 5 & $12 \cdot \mathrm{C}$ & $\mathrm{NI}$ & $95 \%$ & NO LOH & NOLOH & $93 \%$ & $82 \%$ & NOLOH & NI & NOLOH & $\mathrm{NO} L O H$ & NI & $77 \%$ & $84 \%$ & NI & NOLOH \\
\hline 6 & $12 \cdot R$ & NO LOH & $\mathrm{NOLOH}$ & NO LOH & $75 \%$ & $\mathrm{NO} \mathrm{LOH}$ & NOLOH & NOLOH & NOLOH & $\mathrm{NO} \mathrm{LOH}$ & NO LOH & NO LOH & $\mathrm{NO} \mathrm{LOH}$ & $\mathrm{NO} \mathrm{LOH}$ & $\mathrm{NO}$ LOH & NO LOH \\
\hline 6 & $12 \cdot R$ & NO LOH & NOLOH & NOLOH & $69 \%$ & NO LOH & NOLOH & NOLOH & NO LOH & $\mathrm{NO} \mathrm{LOH}$ & $\mathrm{NO}$ LOH & NO LOH & NO LOH & $\mathrm{NO} \mathrm{LOH}$ & NO LOH & NO LOH \\
\hline 7 & 12.D & NI & $\mathrm{NI}$ & $70 \%$ & NI & $94 \%$ & NI & NO LOH & NO LOH & NO LOH & $\mathrm{NO}$ LOH & NO LOH & $83 \%$ & $\mathrm{NO} \mathrm{LOH}$ & $\mathrm{NO} \mathrm{LOH}$ & NO LOH \\
\hline 8 & NO MUT & $\mathrm{NO}$ LOH & $\mathrm{NO}$ LOH & $\mathrm{NI}$ & NO LOH & $\mathrm{NOLOH}$ & NO LOH & $94 \%$ & $\mathrm{NI}$ & $\mathrm{NO}$ LOH & $\mathrm{NOLOH}$ & NO LOH & $\mathrm{NI}$ & NO LOH & $\mathrm{NO}$ LOH & NO LOH \\
\hline 9 & $12 \cdot R$ & $97 \%$ & $\mathrm{NI}$ & $88 \%$ & NOLOH & NI & $80 \%$ & NOLOH & NOLOH & NOLOH & NO LOH & NO LOH & NO LOH & $\mathrm{NO} \mathrm{LOH}$ & NI & NO LOH \\
\hline 10 & NOMUT & $\mathrm{NOLOH}$ & NOLOH & $83 \%$ & NOLOH & NO LOH & NOLOH & NOLOH & $60 \%$ & $\mathrm{NO} L O H$ & NOLOH & NOLOH & NOLOH & $\mathrm{NO} \mathrm{LOH}$ & $\mathrm{NO}$ LOH & NOLOH \\
\hline 10 & NO MUT & $\mathrm{NO} \mathrm{LOH}$ & $\mathrm{NOLOH}$ & $67 \%$ & NO LOH & NO LOH & NOLOH & $54 \%$ & $52 \%$ & $\mathrm{NO} \mathrm{LOH}$ & $\mathrm{NO}$ LOH & NO LOH & NOLOH & $\mathrm{NO} \mathrm{LOH}$ & $\mathrm{NO}$ LOH & NO LOH \\
\hline 11 & 12.D & NO LOH & NOLOH & $60 \%$ & $72 \%$ & NO LOH & NO LOH & NI & $\mathrm{NO}$ LOH & $49 \%$ & $81 \%$ & NO LOH & NO LOH & $\mathrm{NO}$ LOH & $\mathrm{NO} \mathrm{LOH}$ & $53 \%$ \\
\hline 11 & 12-D & $\mathrm{NOLOH}$ & NOLOH & $56 \%$ & $60 \%$ & NO LOH & NOLOH & $\mathrm{NI}$ & $\mathrm{NOLOH}$ & $\mathrm{NOLOH}$ & $85 \%$ & NO LOH & NOLOH & $\mathrm{NO} \mathrm{LOH}$ & NOLOH & $51 \%$ \\
\hline 12 & NO MUT & $\mathrm{NO}$ LOH & $\mathrm{NOLOH}$ & $\mathrm{NI}$ & $98 \%$ & NO LOH & NOLOH & $\mathrm{NI}$ & NOLOH & NI & $\mathrm{NI}$ & NO LOH & NOLOH & $70 \%$ & $\mathrm{NO} \mathrm{LOH}$ & NO LOH \\
\hline 13 & 12.D & $\mathrm{NI}$ & $58 \%$ & NO LOH & $\mathrm{NO} \mathrm{LOH}$ & NO LOH & NOLOH & NI & $98 \%$ & $\mathrm{NO} \mathrm{LOH}$ & NOLOH & NO LOH & $51 \%$ & $59 \%$ & NO LOH & NI \\
\hline 14 & NO MUT & $\mathrm{NI}$ & $\mathrm{NOLOH}$ & NO LOH & NO LOH & $\mathrm{NI}$ & NO LOH & NOLOH & $\mathrm{NOLOH}$ & $51 \%$ & $\mathrm{NO}$ LOH & $\mathrm{NI}$ & $\mathrm{NO} \mathrm{LOH}$ & $67 \%$ & NO LOH & $\mathrm{NI}$ \\
\hline 15 & $12 \mathrm{D}$ & $\mathrm{NO}$ LOH & NI & NO LOH & NI & NO LOH & NOLOH & $78 \%$ & NOLOH & $\mathrm{NOLOH}$ & $\mathrm{NO}$ LOH & NO LOH & $\mathrm{NO} \mathrm{LOH}$ & $71 \%$ & $\mathrm{NI}$ & $\mathrm{NI}$ \\
\hline 15 & $12 D$ & NO LOH & $\mathrm{NI}$ & NOLOH & $\mathrm{NI}$ & NO LOH & NOLOH & $83 \%$ & $\mathrm{NOLOH}$ & $\mathrm{NOLOH}$ & $\mathrm{NO}$ LOH & NO LOH & $\mathrm{NO} \mathrm{LOH}$ & $99 \%$ & $\mathrm{NI}$ & $\mathrm{NI}$ \\
\hline 16 & $12 \cdot \mathrm{V}$ & NO LOH & NI & $83 \%$ & NOLOH & $\mathrm{NI}$ & NI & $\mathrm{NO} \mathrm{LOH}$ & NI & $\mathrm{NO} \mathrm{LOH}$ & NO LOH & NO LOH & NOLOH & $95 \%$ & NO LOH & NO LOH \\
\hline 16 & $12 \cdot \mathrm{V}$ & NO LOH & $\mathrm{NI}$ & $91 \%$ & $73 \%$ & $\mathrm{NI}$ & NI & NO LOH & $\mathrm{NI}$ & $\mathrm{NO}$ LOH & NO LOH & NO LOH & $70 \%$ & $62 \%$ & NO LOH & NO LOH \\
\hline 17 & $12 \cdot R$ & NO LOH & $\mathrm{NO}$ LOH & NOLOH & $\mathrm{NO} \mathrm{LOH}$ & NO LOH & NOLOH & NOLOH & $\mathrm{NOLOH}$ & $\mathrm{NOLOH}$ & $\mathrm{NO}$ LOH & NO LOH & $\mathrm{NO} \mathrm{LOH}$ & $\mathrm{NO} \mathrm{LOH}$ & $\mathrm{NO} L O H$ & NO LOH \\
\hline 17 & $12 \cdot R$ & NO LOH & NO LOH & NO LOH & NOLOH & NO LOH & NOLOH & NOLOH & $\mathrm{NO} \mathrm{LOH}$ & NO LOH & $\mathrm{NO}$ LOH & NO LOH & NO LOH & $\mathrm{NO}$ LOH & $\mathrm{NO}$ LOH & NO LOH \\
\hline 18 & $12 \cdot R$ & $99 \%$ & NOLOH & NO LOH & $91 \%$ & NOLOH & NO LOH & $\mathrm{NO}$ LOH & NO LOH & $\mathrm{NI}$ & NOLOH & NO LOH & NO LOH & $67 \%$ & NOLOH & $\mathrm{NI}$ \\
\hline 19 & $12 . V$ & $\mathrm{NO} \mathrm{LOH}$ & $\mathrm{NI}$ & $\mathrm{NO} \mathrm{LOH}$ & $\mathrm{NO} \mathrm{LOH}$ & NOLOH & $\mathrm{NOLOH}$ & $\mathrm{NOLOH}$ & $\mathrm{NI}$ & $\mathrm{NO} \mathrm{LOH}$ & $\mathrm{NOLOH}$ & $\mathrm{NO}$ LOH & $\mathrm{NOLOH}$ & $\mathrm{NO} \mathrm{LOH}$ & $\mathrm{NI}$ & $\mathrm{NOLOH}$ \\
\hline 20 & $12 \cdot R$ & $\mathrm{NO} \mathrm{LOH}$ & $\mathrm{NOLOH}$ & $81 \%$ & $\mathrm{NO}$ LOH & $\mathrm{NI}$ & $92 \%$ & $\mathrm{NO} \mathrm{LOH}$ & $\mathrm{NO} L \mathrm{LH}$ & $\mathrm{NI}$ & $95 \%$ & $68 \%$ & $73 \%$ & $99 \%$ & $\mathrm{NO} \mathrm{LOH}$ & $\mathrm{NO}$ LOH \\
\hline 21 & NOMUT & NI & $\mathrm{NOLOH}$ & $\mathrm{NO} \mathrm{LOH}$ & $\mathrm{NO} \mathrm{LOH}$ & NOLOH & $\mathrm{NOLOH}$ & $\mathrm{NOLOH}$ & $54 \%$ & $\mathrm{NO} \mathrm{LOH}$ & $\mathrm{NO}$ LOH & NOLOH & $\mathrm{NO}$ LOH & $\mathrm{NOLOH}$ & NO LOH & $\mathrm{NO}$ LOH \\
\hline 22 & $12 \mathrm{~V}$ & $\mathrm{NI}$ & $58 \%$ & NOLOH & $53 \%$ & NOLOH & $62 \%$ & $\mathrm{NI}$ & $\mathrm{NI}$ & $\mathrm{NO} \mathrm{LOH}$ & NI & $\mathrm{NOLOH}$ & NI & $\mathrm{NOLOH}$ & $\mathrm{NOLOH}$ & $\mathrm{NOLOH}$ \\
\hline 22 & $12 \mathrm{~V}$ & NI & $62 \%$ & $92 \%$ & NO LOH & NO LOH & $71 \%$ & NI & $\mathrm{NI}$ & $\mathrm{NO} \mathrm{LOH}$ & NI & NO LOH & $\mathrm{NI}$ & $\mathrm{NO} L \mathrm{LH}$ & NO LOH & $\mathrm{NO}$ LOH \\
\hline 23 & NO MUT & NI & $\mathrm{NOLOH}$ & $\mathrm{NO} \mathrm{LOH}$ & $\mathrm{NI}$ & NO LOH & $\mathrm{NO}$ LOH & $\mathrm{NI}$ & $\mathrm{NO}$ LOH & $\mathrm{NI}$ & NO LOH & NO LOH & $\mathrm{NI}$ & $90 \%$ & $\mathrm{NI}$ & $\mathrm{NI}$ \\
\hline 23 & NO MUT & $\mathrm{NI}$ & $\mathrm{NO}$ LOH & NO LOH & NI & NOLOH & $\mathrm{NO}$ LOH & $\mathrm{NI}$ & $\mathrm{NO}$ LOH & $\mathrm{NI}$ & $\mathrm{NO} \mathrm{LOH}$ & NOLOH & NI & $79 \%$ & NI & $\mathrm{NI}$ \\
\hline 24 & NO MUT & $\mathrm{NO}$ LOH & $90 \%$ & NO LOH & $\mathrm{NI}$ & NO LOH & NO LOH & $89 \%$ & $84 \%$ & $\mathrm{NO} \mathrm{LOH}$ & NO LOH & $61 \%$ & $\mathrm{NI}$ & $\mathrm{NO} \mathrm{LOH}$ & $50 \%$ & $\mathrm{NO}$ LOH \\
\hline 25 & NO MUT & $75 \%$ & $\mathrm{NI}$ & NO LOH & $\mathrm{NO} L O H$ & NOLOH & NO LOH & $\mathrm{NO} \mathrm{LOH}$ & $\mathrm{NO} L O H$ & NO LOH & $\mathrm{NO} \mathrm{LOH}$ & $\mathrm{NOLOH}$ & $\mathrm{NI}$ & $\mathrm{NOLOH}$ & $\mathrm{NO}$ LOH & $\mathrm{NI}$ \\
\hline 26 & $12 \cdot R$ & $\mathrm{NOLOH}$ & $\mathrm{NO}$ LOH & NI & $\mathrm{NO}$ LOH & $\mathrm{NOLOH}$ & NI & $\mathrm{NO} \mathrm{LOH}$ & $\mathrm{NO}$ LOH & NI & $\mathrm{NI}$ & NO LOH & $\mathrm{NO}$ LOH & $\mathrm{NI}$ & NOLOH & $\mathrm{NO}$ LOH \\
\hline 27 & NOMUT & $\mathrm{NOLOH}$ & $\mathrm{NOLOH}$ & NO LOH & $\mathrm{NO}$ LOH & $56 \%$ & $\mathrm{NOLOH}$ & $\mathrm{NOLOH}$ & NOLOH & $\mathrm{NO}$ LOH & NOLOH & NOLOH & $\mathrm{NOLOH}$ & $\mathrm{NI}$ & NI & $\mathrm{NO}$ LOH \\
\hline 27 & NO MUT & $\mathrm{NO}$ LOH & $\mathrm{NOLOH}$ & $\mathrm{NO}$ LOH & $\mathrm{NO}$ LOH & $49 \%$ & $59 \%$ & $\mathrm{NO} \mathrm{LOH}$ & $\mathrm{NO} L O H$ & NO LOH & NO LOH & NOLOH & $\mathrm{NOLOH}$ & NI & NI & $\mathrm{NO}$ LOH \\
\hline 28 & $12 \cdot R$ & $67 \%$ & $82 \%$ & NO LOH & $\mathrm{NO}$ LOH & $\mathrm{NO} \mathrm{LOH}$ & $\mathrm{NO}$ LOH & $\mathrm{NO}$ LOH & NOLOH & NO LOH & NO LOH & NOLOH & NI & $\mathrm{NOLOH}$ & NOLOH & $\mathrm{NI}$ \\
\hline 29 & NO MUT & $\mathrm{NI}$ & $\mathrm{NO} \mathrm{LOH}$ & $\mathrm{NO}$ LOH & NO LOH & NOLOH & $\mathrm{NO} \mathrm{LOH}$ & $\mathrm{NOLOH}$ & NI & $\mathrm{NO} \mathrm{LOH}$ & NO LOH & NO LOH & NO LOH & $49 \%$ & NO LOH & $\mathrm{NO} \mathrm{LOH}$ \\
\hline 30 & NO MUT & NO LOH & $\mathrm{NO}$ LOH & $\mathrm{NI}$ & $\mathrm{NO}$ LOH & $34 \%$ & $43 \%$ & $\mathrm{NO}$ LOH & NO LOH & $\mathrm{NO}$ LOH & NO LOH & NI & NO LOH & $\mathrm{NOLOH}$ & NO LOH & NI \\
\hline 30 & NO MUT & $\mathrm{NO}$ LOH & $\mathrm{NO}$ LOH & NI & $\mathrm{NO}$ LOH & $55 \%$ & $39 \%$ & $\mathrm{NO} \mathrm{LOH}$ & $\mathrm{NO}$ LOH & $\mathrm{NO}$ LOH & NO LOH & NI & $\mathrm{NO}$ LOH & $\mathrm{NOLOH}$ & NO LOH & NI \\
\hline 31 & NO MUT & $\mathrm{NO}$ LOH & $\mathrm{NOLOH}$ & $\mathrm{NI}$ & $\mathrm{NOLOH}$ & $90 \%$ & $\mathrm{NI}$ & $\mathrm{NO} L O H$ & NO LOH & $\mathrm{NI}$ & NOLOH & NO LOH & $\mathrm{NO}$ LOH & $\mathrm{NI}$ & $\mathrm{NOLOH}$ & $\mathrm{NO} \mathrm{LOH}$ \\
\hline 32 & NO MUT & $\mathrm{NO} \mathrm{LOH}$ & $91 \%$ & $\mathrm{NI}$ & $\mathrm{NO}$ LOH & $\mathrm{NO} \mathrm{LOH}$ & NOLOH & $\mathrm{NI}$ & $\mathrm{NO}$ LOH & $\mathrm{NO} \mathrm{LOH}$ & NO LOH & NOLOH & $\mathrm{NO}$ LOH & $\mathrm{NI}$ & NI & $\mathrm{NOLOH}$ \\
\hline 32 & NO MUT & $\mathrm{NOLOH}$ & $58 \%$ & NI & $\mathrm{NO}$ LOH & $\mathrm{NO}$ LOH & NOLOH & $\mathrm{NI}$ & $\mathrm{NOLOH}$ & $\mathrm{NO}$ LOH & NO LOH & NOLOH & $\mathrm{NOLOH}$ & $\mathrm{NI}$ & NI & $\mathrm{NOLOH}$ \\
\hline 33 & NO MUT & NI & $\mathrm{NOLOH}$ & NO LOH & $\mathrm{NO}$ LOH & $60 \%$ & NO LOH & $\mathrm{NO}$ LOH & $\mathrm{NO}$ LOH & $\mathrm{NO}$ LOH & $87 \%$ & NI & $\mathrm{NO}$ LOH & $99 \%$ & NOLOH & NI \\
\hline 33 & NO MUT & NI & $\mathrm{NOLOH}$ & NO LOH & $\mathrm{NO}$ LOH & NI & $\mathrm{NO}$ LOH & $\mathrm{NO} \mathrm{LOH}$ & NOLOH & $\mathrm{NO}$ LOH & $68 \%$ & NI & $52 \%$ & $\mathrm{NOLOH}$ & NO LOH & NI \\
\hline 34 & NO MUT & $\mathrm{NO} \mathrm{LOH}$ & $\mathrm{NI}$ & NO LOH & $\mathrm{NO}$ LOH & $61 \%$ & $\mathrm{NI}$ & $\mathrm{NO}$ LOH & $\mathrm{NI}$ & $\mathrm{NI}$ & $\mathrm{NO} L O H$ & $\mathrm{NI}$ & $\mathrm{NOLOH}$ & $\mathrm{NI}$ & NOLOH & $\mathrm{NI}$ \\
\hline 34 & NO MUT & $\mathrm{NO}$ LOH & $\mathrm{NI}$ & NO LOH & $\mathrm{NO}$ LOH & $62 \%$ & $\mathrm{NI}$ & $\mathrm{NO}$ LOH & NI & $\mathrm{NI}$ & NO LOH & NI & $\mathrm{NO}$ LOH & $\mathrm{NI}$ & NO LOH & $\mathrm{NI}$ \\
\hline 35 & $12 D$ & $48 \%$ & $47 \%$ & $\mathrm{NI}$ & $\mathrm{NO}$ LOH & $70 \%$ & $84 \%$ & $\mathrm{NI}$ & $\mathrm{NI}$ & $\mathrm{NO}$ LOH & $\mathrm{NI}$ & NI & $\mathrm{NI}$ & $\mathrm{NI}$ & NI & $\mathrm{NI}$ \\
\hline 35 & 12D & $\mathrm{NOLOH}$ & $\mathrm{NOLOH}$ & NI & $\mathrm{NO}$ LOH & $60 \%$ & $53 \%$ & NI & $\mathrm{NI}$ & $\mathrm{NO}$ LOH & NI & NI & $\mathrm{NI}$ & NI & NI & NI \\
\hline 36 & $12 . \mathrm{V}$ & $\mathrm{NO} \mathrm{LOH}$ & NOLOH & $96 \%$ & $89 \%$ & $\mathrm{NI}$ & NO LOH & $\mathrm{NO} \mathrm{LOH}$ & NOLOH & NO LOH & $\mathrm{NI}$ & NO LOH & NO LOH & $\mathrm{NOLOH}$ & NI & $\mathrm{NO}$ LOH \\
\hline 37 & NOMUT & $\mathrm{NO}$ LOH & $\mathrm{NOLOH}$ & $\mathrm{NI}$ & $87 \%$ & NOLOH & NOLOH & $\mathrm{NOLOH}$ & $\mathrm{NOLOH}$ & NO LOH & NI & NOLOH & $\mathrm{NO}$ LOH & NI & NOLOH & $\mathrm{NOLOH}$ \\
\hline 38 & 12-D & $\mathrm{NO} \mathrm{LOH}$ & NI & NO LOH & $\mathrm{NO}$ LOH & $\mathrm{NI}$ & NO LOH & $\mathrm{NO}$ LOH & NI & NO LOH & NO LOH & NI & NO LOH & $\mathrm{NO}$ LOH & NO LOH & $\mathrm{NOLOH}$ \\
\hline 39 & 12-D & NO LOH & NI & NO LOH & $\mathrm{NO}$ LOH & NOLOH & $58 \%$ & $62 \%$ & $\mathrm{NO}$ LOH & $\mathrm{NI}$ & NO LOH & NO LOH & $\mathrm{NO}$ LOH & $\mathrm{NO}$ LOH & NI & $\mathrm{NO}$ LOH \\
\hline 40 & $12 D$ & NOLOH & $\mathrm{NI}$ & $\mathrm{NI}$ & $61 \%$ & NO LOH & NO LOH & $\mathrm{NI}$ & $56 \%$ & NO LOH & $53 \%$ & NOLOH & $\mathrm{NO}$ LOH & $\mathrm{NI}$ & NOLOH & $\mathrm{NO} \mathrm{LOH}$ \\
\hline 40 & $12 \mathrm{D}$ & $\mathrm{NO} \mathrm{LOH}$ & $\mathrm{NI}$ & $\mathrm{NI}$ & $\mathrm{NO}$ LOH & $\mathrm{NO}$ LOH & NO LOH & NI & $51 \%$ & $\mathrm{NO}$ LOH & $\mathrm{NO} L \mathrm{LOH}$ & $56 \%$ & NO LOH & $\mathrm{NI}$ & $\mathrm{NO}$ LOH & $\mathrm{NO} \mathrm{LOH}$ \\
\hline
\end{tabular}


Table 1 Continued

\begin{tabular}{|c|c|c|c|c|c|c|c|c|c|c|c|c|c|c|c|c|}
\hline & & $1 \mathrm{p} 36$ & $1 \mathrm{p} 36$ & $3 p 25$ & $3 p 25$ & $5 q 23$ & $5 q 23$ & $9 p 21$ & $9 p 21$ & $10 q 23$ & $10 q 23$ & $17 p 13$ & $17 p 13$ & $17 q 23$ & $21 q 23$ & $22 q 12$ \\
\hline \multirow{2}{*}{\multicolumn{2}{|c|}{ KRAS }} & D1S & D1S & D3S & D3S & D5S & D5S & D9S & D9S & D10S & D10S & D17S & D17S & D17S & D21S & D22S \\
\hline & & 1193 & 407 & 1539 & 2303 & 592 & 615 & 251 & 254 & 1173 & 520 & 1289 & 974 & 1161 & 144 & 532 \\
\hline \multicolumn{17}{|c|}{ Bile duct } \\
\hline 1 & NO MUT & $\mathrm{NI}$ & NO LOH & NO LOH & NI & NO LOH & NO LOH & NOLOH & $\mathrm{NO}$ LOH & $\mathrm{NI}$ & NO LOH & NI & NO LOH & NO LOH & NI & NO LOH \\
\hline 2 & NO MUT & NI & NO LOH & $\mathrm{NI}$ & NO LOH & NO LOH & NO LOH & NOLOH & NI & NO LOH & $71 \%$ & NO LOH & NI & NO LOH & NO LOH & NO LOH \\
\hline 2 & NO MUT & NI & NO LOH & $\mathrm{NI}$ & NO LOH & NO LOH & NO LOH & NOLOH & NI & NO LOH & $73 \%$ & NO LOH & NI & NO LOH & NO LOH & NO LOH \\
\hline 3 & NO MUT & NO LOH & NI & NO LOH & NO LOH & NO LOH & NO LOH & NI & NO LOH & NO LOH & $\mathrm{NI}$ & $\mathrm{NO} \mathrm{LOH}$ & NI & NO LOH & NI & NI \\
\hline 3 & NO MUT & NO LOH & NI & NO LOH & $\mathrm{NO}$ LOH & NO LOH & $\mathrm{NO} \mathrm{LOH}$ & NI & NOLOH & NO LOH & $\mathrm{NI}$ & $\mathrm{NO} \mathrm{LOH}$ & NI & NO LOH & NI & NI \\
\hline 4 & NO MUT & $55 \%$ & NO LOH & NI & NI & NO LOH & NI & NO LOH & NO LOH & NI & NOLOH & NI & NO LOH & NO LOH & NOLOH & NI \\
\hline 4 & NO MUT & $49 \%$ & NO LOH & $\mathrm{NI}$ & $\mathrm{NI}$ & NO LOH & NI & NO LOH & $52 \%$ & NI & NO LOH & NI & NO LOH & NO LOH & NOLOH & NI \\
\hline 5 & NI & NO LOH & NO LOH & NO LOH & NO LOH & NO LOH & NI & NOLOH & NOLOH & NO LOH & NI & NO LOH & NO LOH & NO LOH & NOLOH & NO LOH \\
\hline 5 & NI & NO LOH & NO LOH & NO LOH & $\mathrm{NO} \mathrm{LOH}$ & NO LOH & NI & NOLOH & NO LOH & NO LOH & NI & $\mathrm{NO} \mathrm{LOH}$ & NO LOH & NO LOH & $\mathrm{NOLOH}$ & NO LOH \\
\hline 6 & NO MUT & NO LOH & NO LOH & NI & NO LOH & NO LOH & NO LOH & NO LOH & NO LOH & NI & NO LOH & NO LOH & NO LOH & NO LOH & NI & NI \\
\hline 7 & NO MUT & NO LOH & NI & $\mathrm{NI}$ & NO LOH & $58 \%$ & NO LOH & NI & NO LOH & NO LOH & NO LOH & NO LOH & NO LOH & NO LOH & NI & $\mathrm{NI}$ \\
\hline 7 & NO MUT & NO LOH & NI & $\mathrm{NI}$ & NO LOH & $58 \%$ & NO LOH & NI & NO LOH & NO LOH & NO LOH & NO LOH & NO LOH & NO LOH & NI & NI \\
\hline 8 & NO MUT & NI & NO LOH & NO LOH & $\mathrm{NI}$ & $79 \%$ & $68 \%$ & NO LOH & $\mathrm{NI}$ & $\mathrm{NI}$ & NO LOH & NO LOH & NO LOH & NI & $\mathrm{NOLOH}$ & NO LOH \\
\hline 8 & NO MUT & $\mathrm{NI}$ & NO LOH & NO LOH & NI & $69 \%$ & $66 \%$ & NO LOH & NI & $\mathrm{NI}$ & NO LOH & NO LOH & NO LOH & NI & NO LOH & NO LOH \\
\hline 9 & NI & NO LOH & NO LOH & NO LOH & NO LOH & NO LOH & $\mathrm{NO}$ LOH & NO LOH & NO LOH & $\mathrm{NO}$ LOH & NO LOH & NO LOH & NI & $\mathrm{NO}$ LOH & $\mathrm{NO}$ LOH & NO LOH \\
\hline 10 & NI & NO LOH & NO LOH & $55 \%$ & NO LOH & NO LOH & NO LOH & NOLOH & NO LOH & NO LOH & NO LOH & $\mathrm{NI}$ & NO LOH & $\mathrm{NO}$ LOH & NI & NO LOH \\
\hline 11 & NO MUT & NO LOH & $56 \%$ & $\mathrm{NI}$ & NO LOH & $\mathrm{NI}$ & $\mathrm{NI}$ & NI & $\mathrm{NO}$ LOH & $\mathrm{NI}$ & $\mathrm{NI}$ & $58 \%$ & NO LOH & NO LOH & $\mathrm{NI}$ & $\mathrm{NI}$ \\
\hline 11 & NO MUT & NO LOH & $56 \%$ & $\mathrm{NI}$ & $\mathrm{NO}$ LOH & $\mathrm{NI}$ & NI & NI & NO LOH & NI & $\mathrm{NI}$ & $77 \%$ & $59 \%$ & NO LOH & NI & NI \\
\hline 12 & NO MUT & NI & NO LOH & NO LOH & NI & $\mathrm{NI}$ & NO LOH & NO LOH & NO LOH & $\mathrm{NI}$ & $\mathrm{NI}$ & $\mathrm{NO}$ LOH & NO LOH & NO LOH & NOLOH & NI \\
\hline 13 & NO MUT & NO LOH & NO LOH & $\mathrm{NI}$ & $62 \%$ & NO LOH & $\mathrm{NO}$ LOH & NI & NO LOH & NO LOH & NOLOH & NO LOH & NO LOH & NI & NO LOH & NO LOH \\
\hline 14 & 12-D & NI & $60 \%$ & NO LOH & $\mathrm{NO}$ LOH & NI & NO LOH & NI & $\mathrm{NO}$ LOH & NO LOH & $\mathrm{NI}$ & NI & $57 \%$ & $\mathrm{NI}$ & $\mathrm{NI}$ & $68 \%$ \\
\hline 14 & NO MUT & $\mathrm{NI}$ & NO LOH & NO LOH & NO LOH & $\mathrm{NI}$ & NO LOH & NI & NO LOH & NO LOH & NI & NI & NO LOH & NI & NI & NO LOH \\
\hline 15 & NO MUT & NO LOH & NO LOH & NO LOH & $\mathrm{NI}$ & $63 \%$ & NO LOH & $\mathrm{NO}$ LOH & $\mathrm{NI}$ & NO LOH & NI & NO LOH & $91 \%$ & NO LOH & NI & NO LOH \\
\hline 15 & NO MUT & NO LOH & NO LOH & $69 \%$ & $\mathrm{NI}$ & $60 \%$ & NO LOH & NO LOH & $\mathrm{NI}$ & NO LOH & $\mathrm{NI}$ & $\mathrm{NO} \mathrm{LOH}$ & $91 \%$ & NO LOH & $\mathrm{NI}$ & NO LOH \\
\hline 16 & NO MUT & NO LOH & NO LOH & NO LOH & NO LOH & $\mathrm{NO}$ LOH & $\mathrm{NI}$ & NI & $\mathrm{NO}$ LOH & NI & NI & NO LOH & NO LOH & NO LOH & $\mathrm{NO}$ LOH & NI \\
\hline 16 & NO MUT & NO LOH & NO LOH & NO LOH & NO LOH & NO LOH & $\mathrm{NI}$ & NI & NO LOH & $\mathrm{NI}$ & $\mathrm{NI}$ & NO LOH & NO LOH & $\mathrm{NO}$ LOH & NO LOH & $\mathrm{NI}$ \\
\hline 17 & NO MUT & NI & NO LOH & NO LOH & NO LOH & $60 \%$ & NI & NOLOH & $\mathrm{NO}$ LOH & NO LOH & NI & NO LOH & NO LOH & NO LOH & $\mathrm{NO}$ LOH & NI \\
\hline 17 & NO MUT & NI & NO LOH & NO LOH & $\mathrm{NO} \mathrm{LOH}$ & $60 \%$ & NI & NOLOH & NO LOH & NO LOH & NI & $\mathrm{NO} \mathrm{LOH}$ & NO LOH & NO LOH & $\mathrm{NO} L O H$ & NI \\
\hline 18 & $12 \cdot V$ & NO LOH & NI & NO LOH & $\mathrm{NI}$ & NO LOH & NO LOH & NI & NOLOH & NO LOH & NI & NO LOH & $99 \%$ & NI & NI & NO LOH \\
\hline 18 & $12 \cdot V$ & NO LOH & NI & NO LOH & NI & NO LOH & NO LOH & NI & $\mathrm{NO} \mathrm{LOH}$ & NO LOH & NI & NO LOH & $97 \%$ & NI & $\mathrm{NI}$ & NO LOH \\
\hline 19 & NO MUT & $\mathrm{NO} \mathrm{LOH}$ & NI & NO LOH & $51 \%$ & $\mathrm{NI}$ & NO LOH & NO LOH & $\mathrm{NO}$ LOH & $\mathrm{NI}$ & NO LOH & NO LOH & NO LOH & $\mathrm{NI}$ & $\mathrm{NO} \mathrm{LOH}$ & NO LOH \\
\hline 19 & NO MUT & NO LOH & NI & NO LOH & $63 \%$ & $\mathrm{NI}$ & NO LOH & NOLOH & $\mathrm{NO}$ LOH & $\mathrm{NI}$ & NO LOH & NO LOH & NO LOH & NI & NOLOH & NO LOH \\
\hline 20 & NI & NO LOH & NO LOH & NO LOH & NI & NO LOH & $\mathrm{NO} \mathrm{LOH}$ & NOLOH & NI & NO LOH & NO LOH & NO LOH & NO LOH & NO LOH & $73 \%$ & NI \\
\hline 20 & NI & NO LOH & NO LOH & $\mathrm{NO}$ LOH & NI & NO LOH & $\mathrm{NO}$ LOH & NOLOH & NI & NOLOH & NO LOH & NO LOH & NO LOH & $\mathrm{NO}$ LOH & $71 \%$ & $\mathrm{NI}$ \\
\hline 21 & NO MUT & $53 \%$ & $45 \%$ & NO LOH & NO LOH & $\mathrm{NI}$ & NO LOH & NOLOH & NI & $68 \%$ & NI & $46 \%$ & $58 \%$ & NO LOH & $\mathrm{NO}$ LOH & NO LOH \\
\hline 21 & NO MUT & $84 \%$ & $47 \%$ & NO LOH & NO LOH & $\mathrm{NI}$ & NO LOH & $\mathrm{NO}$ LOH & NI & $99 \%$ & $\mathrm{NI}$ & $67 \%$ & $89 \%$ & NO LOH & $\mathrm{NO}$ LOH & NO LOH \\
\hline
\end{tabular}

derived from equivalent aliquots of the microdissected sample, markers exhibiting more extreme degrees of mutational content were acquired before markers showing lesser degree of mutational content. For each patient, the unique temporal profile of mutation acquisition could be determined with a representative example shown in Figure 3.

In the malignant cytology group, 4/10 patients had histology follow-up and carcinoma was confirmed in these cases. Out of 20 in the atypical cytology group, nine had histologic follow-up with carcinoma in 4/9 specimens. Out of 10 in the benign cytology cases, four had histology follow-up with $3 / 4$ had a benign diagnosis.

\section{Conclusions}

The determination of the constellation of cancer related changes in a neoplastic specimen has received great interest due to genome wide techniques, such as DNA microarrays, comparative genomic hybridization and proteomic chip technology. ${ }^{24-26}$ Timing of mutation acquisition may rightly be expected to exert profound effects on cancer biology and may also influence responsiveness to the treatment. ${ }^{10,11}$

Knowing that cancer evolves through a temporal process of mutation acquisition raises the question whether this sequence can be determined in clinical specimens. Mutations that are causally responsible for a significant increase in neoplastic behavior should be reflected within individual cells and across the tumor by the degree to which they induce clonal expansion of more aggressive tumor cells. We have shown previously that mutations present in virtually all clonal cells are likely to have been acquired early in tumorigenesis and may be largely responsible for phenotypic expression and cancer behavior. ${ }^{27}$ In contrast, mutations that are focally 


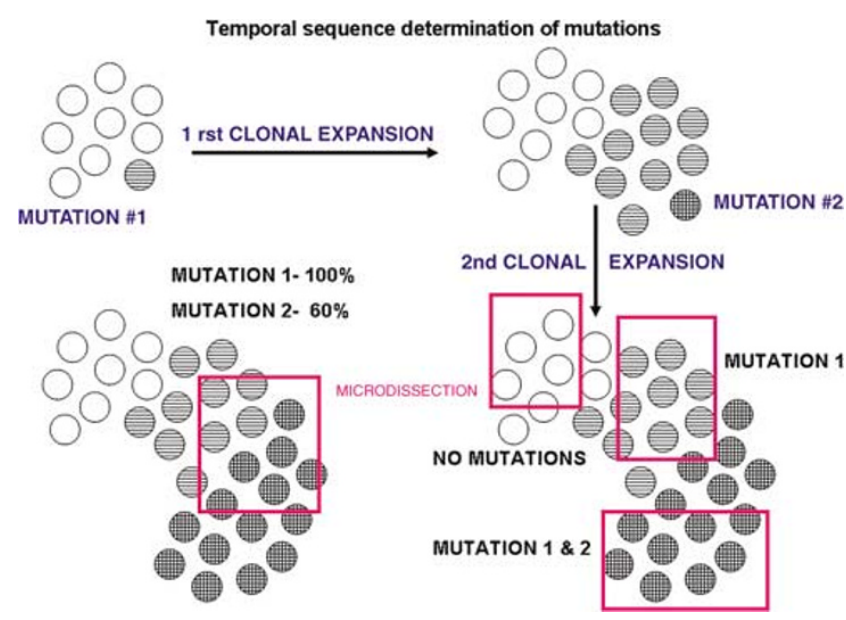

Figure 3 The temporal profile of sequential mutation acquisition can be represented by a schematic diagram highlighting the order to mutation accumulation. Mutations acquired early are present in multiple microdissection targets and at a higher proportion of affected cells. Mutations acquired later in temporal sequence tend to be more limited in topographic extent and at a relative lower percentage of affected cells. The temporal profile demonstrates the diversity that exists between different patients with the same histologic form of cancer.

distributed likely to have arrived later in cancer evolution and may be expected to contribute minimally to the overall phenotype of that neoplasm. ${ }^{24}$

We have shown that pancreatic and bile duct cancers share allelic imbalance damage for a subset of mutational markers (Table 1). The descending frequency of detectable mutational involvement in pancreatic cytology was K-ras-2 point mutation (58\%), 3p25-26 and 17q21 (35\%), 5q23 (33\%), 1 p36 (28\%), followed by the remaining molecular markers. K-ras-2 point mutation occurred in all but 1 malignant case and was associated with abundant coexisting acquired mutational damage. The descending frequency of detectable mutational involvement in bile duct cytology was $17 \mathrm{p} 13,1 \mathrm{p} 36$, 3p25-26, 5q23 followed by remaining markers. While there was overlap in the spectrum of mutational markers, the temporal profile was significantly different $(P<0.001)$. This supports the concept that tumorigenesis is not a random process, but one guided by factors that are as yet not fully understood.

In order to address the need for more definitive diagnosis in cytology, the correlation of molecular information appears to be a most productive option to undertake. ${ }^{28-30}$ This information however must be presented in the context of microscopic findings that is essential to appropriately integrate molecular information. Mutational profiling is best carried out after microscopic evaluation of all available specimens to define the optimal targets for correlative molecular analysis. The microdissection platform, where small numbers of cells are obtained and then subdivided for broad panel analysis offers an effective means to generate significant amounts of data from limited size specimens. By utilizing quantitative means applied to multiple microdissection targets, the temporal profile can be developed generating patient specific information at the individual patient level. ${ }^{31-35}$ In conclusion, we believed that the determination of sequential timing of mutational damage in cytologic material could be incorporated in the work-up and help to make more definitive diagnosis of malignancy in pancreatobiliary cytology specimens

\section{References}

1 Layfield LJ, Cramer H. Fine-needle aspiration cytology of intraductal papillary-mucinous tumors: a retrospective analysis. Diagn Cytopathol 2005;32:16-20.

2 Bartoletti R, Dal Canto M, Cai T, et al. Early diagnosis and monitoring of superficial transitional cell carcinoma by microsatellite analysis on urine sediment. Oncol Rep 2005;13:531-537.

3 Acha A, Ruesga MT, Rodriguez MJ, et al. Applications of the oral scraped (exfoliative) cytology in oral cancer and precancer. Med Oral Patol Oral Cir Bucal 2005; 10:95-102.

4 Lal A, Bourtsos EP, DeFrias DV, et al. Microcystic adenoma of the pancreas: clinical, radiologic, and cytologic features. Cancer 2004;102:288-294.

5 Recine M, Kaw M, Evans DB, et al. Fine-needle aspiration cytology of mucinous tumors of the pancreas. Cancer 2004;102:92-99.

6 Basir Z, Pello N, Dayer AM, et al. Accuracy of cytologic interpretation of pancreatic neoplasms by fine needle aspiration and pancreatic duct brushings. Acta Cytol 2003:47:733-738.

7 Moolgavkar SH, Luebeck EG. Multistage carcinogenesis and the incidence of human cancer. Genes Chromosomes Cancer 2003;38:302-306.

8 Weiss RA. Multistage carcinogenesis. Br J Cancer 2004; 91:1981-1982.

9 Owens DM, Wei S, Smart RC. A multihit, multistage model of chemical carcinogenesis. Carcinogenesis 1999;20:1837-1844.

10 Mohan D, Finkelstein SD, Swalsky PA, et al. Microdissection genotyping of gliomas: therapeutic and prognostic considerations. Mod Pathol 2004;17: 1346-1358.

11 McLendon R, Swalsky P, Finkelstein S. Determination and significance of the timing of $1 p / 19 q$ allelic loss in relation to aggressive mutations (9p, 10q, 17p) in gliomas. J Neuropathol Exp Neurol 2004;63: 551.

12 Chow M, Rubin H. Clonal dynamics of progressive neoplastic transformation. Proc Natl Acad Sci USA 1999;96:6976-6981.

13 Losi L, Baisse B, Bouzourene H, et al. Evolution of intratumoral genetic heterogeneity during colorectal cancer progression. Carcinogenesis 2005;26: 916-922.

14 Breivik J. The evolutionary origin of genetic instability in cancer development. Semin Cancer Biol 2005;15: 51-60.

15 Cahill DP, Kinzler KW, Vogelstein B, et al. Genetic instability and Darwinian selection in tumours. Trends Cell Biol 1999;9:M57-M60. 
16 Lengauer C, Kinzler KW, Vogelstein B. Genetic instabilities in human cancers. Nature 1998;396:643-649.

17 Finkelstein SD, Marsh W, Demetris AJ, et al. Microdissection-based allelotyping discriminates de novo tumor from intrahepatic spread in hepatocellular carcinoma. Hepatology 2003;37:871-879.

18 Ohori NP, Khalid A, Etemad B, et al. Multiple loss of heterozygosity without K-ras mutation identified by molecular analysis on fine-needle aspiration cytology specimen of acinar cell carcinoma of pancreas. Diagn Cytopathol 2002;27:42-46.

19 Khalid A, Pal R, Sasatomi E, et al. Use of microsatellite marker loss of heterozygosity in accurate diagnosis of pancreaticobiliary malignancy from brush cytology samples. Gut 2004;53:1860-1865.

20 Luttges J, Kloppel G. Update on the pathology and genetics of exocrine pancreatic tumors with ductal phenotype: precursor lesions and new tumor entities. Dig Dis 2001;19:15-23.

21 Cowgill SM, Muscarella P. The genetics of pancreatic cancer. Am J Surg Pathol 2003;186:279-286.

22 Yeo TP, Hruban RH, Leach SD, et al. Pancreatic cancer. Curr Probl Cancer 2002;26:176-275.

23 Hunt JL, Finkelstein SD. Microdissection techniques for molecular testing in surgical pathology. Arch Pathol Lab Med 2004;128:1372-1378.

24 Hardiman G. Microarray platforms-comparisons and contrasts. Pharmacogenomics 2004;5:487-502.

25 Rickert CH, Paulus W. Comparative genomic hybridization in central and peripheral nervous system tumors of childhood and adolescence. J Neuropathol Exp Neurol 2004;63:399-417.

26 Wulfkuhle J, Espina V, Liotta L, et al. Genomic and proteomic technologies for individualisation and improvement of cancer treatment. Eur J Cancer 2004;40:2623-2632.

27 Sasatomi E, Finkelstein SD, Woods JD, et al. Comparison of accumulated allele loss between primary tumor and lymph node metastasis in stage II non-small cell lung carcinoma: implications for the timing of lymph node metastasis and prognostic value. Cancer Res 2002;62:2681-2689.

28 Khalid A, McGrath K, Zahid M, et al. The role of pancreatic cyst fluid molecular analysis in predicting cyst pathology. Clin Gastroenterol Hepatol 2005;3: 967-973.

29 Basir Z, Pello N, Dayer AM, et al. Accuracy of cytologic interpretation of pancreatic neoplasms by fine needle aspiration and pancreatic duct brushings. Acta Cytol 2003;47:733-738.

30 Maire F, Couvelard A, Hammel P, et al. Intraductal papillary mucinous tumors of the pancreas: the preoperative value of cytologic and histopathologic diagnosis. Gastrointest Endosc 2003;58:701-706.

31 Vogelstein B, Kinzler KW. Cancer genes and the pathways they control. Nat Med 2004;10:789-799.

32 Arends JW. Molecular interactions in the Vogelstein model of colorectal carcinoma. J Pathol 2000;190: 412-416.

33 Yokota J, Kohno T. Molecular footprints of human lung cancer progression. Cancer Sci 2004;95:197-204.

34 Ingvarsson S. Genetics of breast cancer. Drugs Today (Barcelona) 2004;40:991-1002.

35 Pan H, Califano J, Ponte JF, et al. Loss of heterozygosity patterns provide fingerprints for genetic heterogeneity in multistep cancer progression of tobacco smokeinduced non-small cell lung cancer. Cancer Res 2005; 65:1664-1669. 